\title{
Upfront surgery vs. neoadjuvant therapy for resectable pancreatic cancer: a narrative review of available evidence
}

\author{
Chathura B. B. Ratnayake ${ }^{1}$, Keith J. Roberts ${ }^{2}$, Sanjay Pandanaboyana ${ }^{3,4}$ \\ ${ }^{1}$ Department of Surgery, Faculty of Medical and Health Sciences, University of Auckland, Auckland, New Zealand; ${ }^{2}$ Department of Hepatobiliary, Pancreatic \\ and Transplant Surgery, Queen Elizabeth Hospital, Birmingham, UK; ${ }^{3}$ Department of Hepatobiliary, Pancreatic and Transplant Surgery, Department of \\ Surgery, Freeman Hospital, Newcastle upon Tyne, Tyne and Wear, UK; ${ }^{4}$ Population Health Sciences Institute, Newcastle University, Newcastle, UK \\ Contributions: (I) Conception and design: CBB Ratnayake, S Pandanaboyana; (II) Administrative support: All authors; (III) Provision of study \\ materials or patients: CBB Ratnayake, S Pandanaboyana; (IV) Collection and assembly of data: CBB Ratnayake, S Pandanaboyana; (V) Data analysis \\ and interpretation: All authors; (VI) Manuscript writing: All authors; (VII) Final approval of manuscript: All authors. \\ Correspondence to: Sanjay Pandanaboyana, MS, FRCS (Edin), MPhil. Consultant Surgeon, HPB and Transplant Unit, Freeman Hospital, Newcastle \\ upon Tyne, UK. Email: S.pandanaboyana@nhs.net.
}

Background and Objective: Though the use of neoadjuvant therapy (NAT) is increasing in the setting of borderline resectable (BRPC) and locally advance pancreatic cancer (LAPC), the role of NAT in resectable pancreatic cancer (RPC) remains uncertain.

Methods: This is a narrative review, summarising the contemporary evidence and emerging studies comparing neoadjuvant therapy to upfront resection and adjuvant therapy in RPC.

Key and Content and Findings: Upfront resection followed by adjuvant chemotherapy is currently the standard of care for RPC. Though BRPC and LAPC have reported significant overall survival benefits with NAT, those results have yet to be translated to RPC. Downstaging is only reported in a small proportion of patients who receive NAT; most have stable disease and a small number have progression. Preliminary trial data have largely been consistent with that observed in the past whereby a modest improvement in R0 resection rates and pathological findings is observed with NAT, however rates of distant recurrence and overall survival remain similar to upfront resection. A significant proportion further fail to achieve resection due to the side effects, deconditioning and delays to surgery. Most international recommendations have been guided by non-randomised data sets and long-term data from emerging phase III trials are yet to be published.

Conclusions: Although we have observed improved R0 resection rates with NAT, this has yet to translate to a robust improvement in overall survival. Concerns regarding delays to resection, and limited response to NAT remain a topic of ongoing investigation.

Keywords: Pancreatic cancer; neoadjuvant therapy; upfront surgery; outcomes

Submitted Nov 26, 2021. Accepted for publication Feb 11, 2022.

doi: 10.21037/cco-21-161

View this article at: https://dx.doi.org/10.21037/cco-21-161

\section{Introduction}

Pancreatic cancer is projected to become a leading cause of cancer related mortality in the next decade (1), contributed by a rising incidence, the lack of effective screening, and a limited improvement in treatment strategies (2). Indeed, the five-year survival is now approaching $10 \%$ (3) for all stages of the disease. This modest improvement has been driven largely by the advent of modern chemotherapeutics (4).

Given most patients are asymptomatic or minimally symptomatic during the early stages of pancreatic cancer, patients are often diagnosed with metastatic or locally advanced disease (3). Only $10-15 \%$ are candidates for curative resection with tumour localised to the gland 
Table 1 Literature search strategy

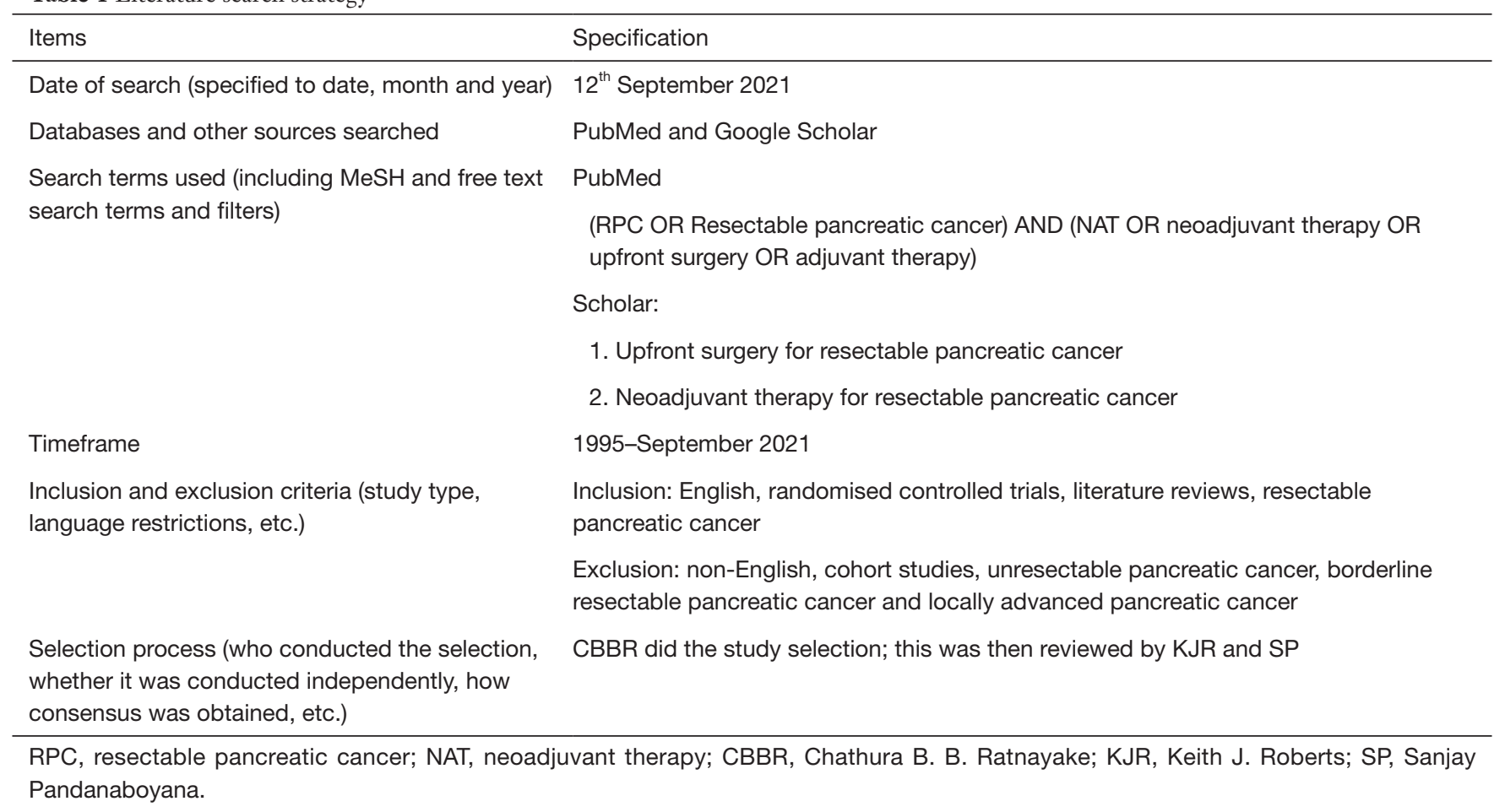

without vascular involvement (3). Adjuvant therapy following resection for resectable pancreatic cancer (RPC) has been shown to prolong survival when compared to surgery alone and has since been the standard of care (5). Despite efforts to improve patient selection and achieve $\mathrm{R} 0$ resection margins, early recurrence is still common. Furthermore more than a third of patients fail to receive adjuvant therapy as a consequence of comorbidities, postoperative complications, and early metastases (6).

The use of neoadjuvant therapy (NAT) for RPC has been proposed as an additional strategy to improve survival following resection. Though the use of NAT in borderline resectable (BRPC) and locally advanced pancreatic cancer (LAPC) is being increasingly accepted (7), the role of NAT in RPC remains uncertain. This narrative review aimed to summarise the current literature on the use of NAT vs. upfront resection and adjuvant therapy in RPC. We present the following article in accordance with the Narrative Review reporting checklist (available at https:// cco.amegroups.com/article/view/10.21037/cco-21-161/rc).

\section{Methods}

Articles were retrieved through the systematic search of two literature databases: PubMed and Google Scholar on the $12^{\text {th }}$ September 2021. Included in the review were English, randomised controlled trials (RCTs) or meta-analyses comparing upfront surgery to NAT for RPC published between January 1995 and September 2021. If articles did not specifically mention RPC they were excluded. The specific search strategy for this narrative review is summarised in Table 1.

\section{Results}

\section{Definitions for non-metastatic pancreatic cancer}

Definitions for staging and resectability have been repeatedly updated as our understanding of the underlying disease process and clinical observations have expanded. Historically, tumors within the limits of the pancreas without contact or involvement of surrounding vascular structures was the defining feature of RPC (8-10). As the importance of a microscopically clear, R0 resection margin in achieving durable long-term survival became clear, the terms BRPC and LAPC were established to denote tumors at a high risk of positive margins. Consequently, the National Comprehensive Cancer Network (NCCN) adopted the first widely accepted BRPC definition (11). 
Table 2 International guideline definitions for resectable pancreatic cancer on computed tomography criteria

\begin{tabular}{lll}
\hline Guideline author & Celiac Axis/SMA/CHA & SMV/PV \\
\hline MD Anderson Center (12,13) & No contact/involvement & No occlusion but includes contact/involvement \\
AHPBA/SSO/SSAT (14) & No contact/involvement & No contact/involvement \\
NCCN (15) & No contact/involvement & $\begin{array}{l}\text { No contact/involvement or } \leq 180-\text { degree contact/involvement without } \\
\text { vein contour irregularity }\end{array}$ \\
Alliance (16) & No contact/involvement & No occlusion but includes contact/involvement <180 degrees
\end{tabular}

American Hepato-Pancreato-Biliary Association/Society of Surgical Oncology/Society for Surgery of the Alimentary Tract (AHPBA/SSO/ SSAT) (14), the National Comprehensive Cancer Network (NCCN). SMA, superior mesenteric artery; CHA, common hepatic artery; SMV, superior mesenteric vein; PV, portal vein.

Currently there are four internationally recognised definitions for RPC: MD Anderson (12,13), American Hepato-Pancreato-Biliary Association/Society of Surgical Oncology/Society for Surgery of the Alimentary Tract (AHPBA/SSO/SSAT) (14), NCCN 2016 update (15), and the Alliance definitions (16). A summary of their definitions for RPC with respect to vessel involvement are summarised in Table 2.

There is consensus between definitions regarding the importance of no arterial involvement in defining RPC, however there is obvious uncertainty with respect to the degree of portal vein/superior mesenteric vein (PV/SMV) involvement. The AHPBA/SSO/SSAT (14) definition is strict, and RPC is denoted by the lack of any tumour involvement of SMV/PV when compared to the MD Anderson $(12,13)$ definition; including all involvement that is not formally occlusive (Table 2). A failure to standardise these definitions has resulted in lack of clarity and questions regarding inter-study reliability. Indeed, Assifi et al reported up to nearly $40 \%$ of patients diagnosed as BRPC using the AHPBA/SSO/SSAT definitions could be reclassified as RPC (17). The contribution of these differences in definitions to the variances in outcomes observed between published international cohorts is unclear but puts into question the translatability and comparability of historical findings.

\section{Recurrence patterns}

Despite improved efforts to achieve R0 resection, disease control is often hampered by early recurrence and treatment failure. Recurrence after pancreatic resection is still frequent and is seen in approximately $20 \%$ of patients within the first 6 months and nearly $40 \%$ within the first 12 months (18). Locoregional recurrence is thought to be primarily contributed by poor tumor differentiation and resection margin status $(19,20)$, whereas tumor diameter, perineural invasion, and preoperative CA19-9 elevation are risk factors for distant recurrence (21). Distant recurrence is often encountered in the liver, lungs and peritoneum (7). It is hypothesised that this observation is due in part to micro metastatic disease that may be present at the time of presentation, undetectable by preoperative clinical imaging (22). NAT has been proposed as a method to achieve micro metastatic disease control and thereby improve rates of recurrence. In a recent meta-analysis of 26 studies comparing upfront surgery $v s$. NAT for PDAC, investigators showed an improvement in locoregional disease control for BRPC, however rates of distant disease recurrence remained unchanged (7). The weighted rate of locoregional and distant recurrence for RPC undergoing upfront resection was $12 \%$ and $37 \%$ respectively at approximately 3 years, however, the study was limited by its ability to compare upfront surgery $v$ s. NAT for RPC due to a paucity of quantitative data. Indeed, only $23 \%$ of patients in the NAT cohort were RPC and the focus in recent trials have been largely BRPC and LAPC.

\section{Upfront resection and adjuvant therapy vs. neoadjuvant therapy}

Upfront resection and adjuvant chemotherapy has been the standard of care for well over a decade. This comes following the results of the CONKO-001 RCT in 2007 (5), where 368 patients were randomised to postoperative adjuvant gemcitabine or observation for 6 months. An improvement in overall survival was observed in those treated with Gemcitabine (22.8 vs. 20.2 months). In combination with the results of the ESPAC-1 trial in 2004 (23), guidelines and recommendations were subsequently transformed. In the intervening years, 
subsequent trials including the ESPAC-4 (24) and PRODIGE-24 trials (4) confirmed the added benefit of multi-agent chemotherapy and the superiority of the FOLFIRINOX (folinic acid, 5-fluorouracil, irinotecan, and oxaliplatin) regimens to those with gemcitabine respectively. FOLFIRINOX is now the recommended first-line adjuvant chemotherapy in those with good postoperative functional status and gemcitabine/capecitabine is generally reserved for those for whom FOLFIRINOX is not an option (25).

Though NAT is gaining interest into the treatment of BRPC and LAPC (26), there are concerns that most patients observe a lack of a major response. In a recent retrospective study by Tajima and colleagues (27) comparing NAT $v s$. US for BRPC, only five out of initial 52 patients allocated to the NAT arm experienced a partial response defined as a greater than $30 \%$ reduction of the sum of two perpendicular dimensions on cross-sectional imaging. The vast majority $(86.5 \%)$ had stable disease and two patients (3.9\%) observed progression of disease. Post therapy imaging showed no improvement in tumour size and despite histopathological injury in all tumor cells, no complete response was found. Consequently 5 -year survival was similar between arms (27). Though advances in medical imaging have allowed the use of multimodal techniques in cross-sectional imaging, faster data acquisition and improved image quality, response evaluation relies on radiology experience and observation by the naked eye (28). Despite this, in a recent study of 77 patients undergoing NAT for BRPC, CT was associated with a high inter-observer reliability in determining tumour response grade, and differentiating RPC, BRPC, and unresectable disease after NAT (29).

In a recent phase III trial by the DUTCH group [Preoperative Chemoradiotherapy Versus Immediate Surgery for Resectable and Borderline Resectable Pancreatic Cancer (PREOPANC-1) (30)], 246 BRPC and RPC patients were randomly allocated to NAT and US (119 vs. 127 respectively). R0 resection rates were markedly improved in NAT arm (71\% vs. $40 \%)$ among those that made it to resection. Fewer surgical site infections (SSIs), and an earlier stage of disease was also observed with NAT. However, median overall survival did not differ (16.0 vs. 14.3 months, $\mathrm{P}=0.096)$. Furthermore, although not statistically significant due to the low-powered nature of the study, a trend towards fewer patients making it to surgery was observed with NAT $(72 \%$ vs. $62 \%, \mathrm{P}=0.058)$. These findings were also confirmed in the preliminary results of a recent phase II/III RCT (NEPAFOX) (31). Here, investigators randomised 40 patients with RPC to adjuvant gemcitabine $v s$. neoadjuvant FOLFIRINOX. Though recruitment was abandoned early due to limitations with numbers, only $58 \%$ of patient made it to resection in the NAT arm $v s .79 \%$ in the adjuvant therapy arm and again comparable overall survival was observed. Conversely, in the Pact-15 trial by Reni and colleagues (32), among the 32 RPC receiving NAT, 29 were resected and 19 were event free at one year, higher than those in the two US cohorts (group A: 6/30 and group B: 15/30 respectively). The authors report lower grade 3 toxicities in the NAT cohort, however this was confounded by the fact only 21 of the 32 patients originally randomised received adjuvant therapy (32). Similarly, preliminary data in the four arm Phase II ESPAC-5F trial has shown an improved one-year survival between NAT (77\%) and upfront surgery (40\%) for BRPC. Though similar resection rates were achieved, only $79 \%$ of patients completed NAT and nine out of 51 patients who underwent NAT had serious adverse events. Longterm data is yet to be published (33). Indeed in the SWOG S1505 trial comparing two perioperative chemotherapy regimens, $72 \%$ (73/102) made it resection and only $33 \%$ (24/73) observed a complete or major pathological response on histology (34). An inadequate response or tumour progression rendering an originally resectable tumour, unresectable is a lingering concern.

\section{Limitations in the previous trials}

There is clearly much interest in NAT and a drive towards NAT in RPC. However, there are fundamental methodological issues which affect all trials in this area. Given more than a third of patients fail to receive adjuvant therapy (6), trials randomising patients prior to surgery $v s$. highly selected cohorts randomising after surgery will differ in their outcomes. Further, there are nuances of the patients pathway that are important to understand. Such issues include how obstructive jaundice are handled within treatment pathways.

Historically, trial data comparing NAT vs. upfront surgery have failed to reach recruitment targets and therefore have been underpowered to reach meaningful conclusions $(35,36)$. They have often been supplemented by a number of nonrandomised cohort studies in quantitative reviews, recruiting the vast majority of included patients in these reviews $(37,38)$. In a recent systematic review by Lee et al., 14 articles were included, containing a single RCT. An improvement in overall survival among those who completed NAT and were ultimately resected was observed compared to those 
undergoing upfront surgery. However, this difference did not persist when comparing all those originally in the NAT and Upfront surgery cohorts (38). Similarly, another systematic review in 2019 containing mostly non-randomised data (8/11 studies) showed improved R0 resection rates among those who had NAT however, reported a comparable overall survival (37). This is in contrast to a recent meta-analysis of six RCTs containing both BRPC and RPC showing an improvement in $\mathrm{R} 0$ resection rates and overall survival independent of resectability (39). The study was limited by definitions for resectability, inclusion of largely lowpowered, historical trials and a variety of chemotherapy and radiotherapy techniques.

'Solution bias' describes the desire for novel/attractive treatments to succeed and may be influencing practice and beliefs in RPC. In the case of RPC, where previous pathways have failed to improve outcomes for so long, clinicians and scientists want to adopt new solutions. Simplistically, NAT is attractive when one considers that many patients with RPC ultimately die of cancer recurrence and given that NAT is standard of care for other cancer sites (40-42), the potential benefits of NAT are assumed to translate to those with RPC. Treatment differences between cohorts exacerbating this problem. For example, in every RCT to date, the cohort randomised to receive NAT receives a different regimen to that randomised to receive adjuvant therapy. The NAT invariably comprises more agents and/or more effective agents (for example, NorPACT-1 NAT FOLFIRINOX + adjuvant gem/cap vs. adjuvant gem/cap and/or additional strategies (example PREOPANC NAT gem + radiotherapy + adjuvant gem $v s$. adjuvant gem). There is also evidence of 'cherry picking' data. In the PREOPANC study, for example where neoadjuvant radiotherapy was used, a key reported outcome was a R0 rate of $72 \%$ in the NAT cohort (51/72), vs. $40 \%$ (37/92) in the resection cohort. However, $43 \%$ more patients did not undergo surgery in the NAT cohort than in the upfront surgery cohort and thus those who eventually made it to surgery were perhaps advantaged in some way. Further, in that study there were significantly more patients with worse performance status (WHO ECOG $058.0 \%$ vs. 30.4\% NAT vs. US) and a higher rate of suspicious lymph nodes on preoperative imaging in the upfront surgery cohort (34.6\% vs. $22.7 \%$ ) as well as having a higher CA19-9 level at baseline. Thus, the data from this study may not be generalizable. However, the R0 rate in the NAT that underwent surgery is a key 'take home' message whilst sources of bias or confounding are easily overlooked. The different regimen received by the cohorts in these trials is typically loaded in favour of effectiveness of agents given to the NAT cohort. It is clear that FOLFIRINOX is highly effective in the adjuvant (4) and palliative settings (43), when compared to multi- or single agent based gemcitabine regimens. Thus patients randomised to receive such therapy in the neoadjuvant setting may simply be having benefit of a more effective therapy, than those randomised to receive gemcitabine based adjuvant therapies, and not a benefit from the timing of therapy in relation to surgery. This is an important point. With an upfront surgery cohort, biliary drainage can be avoided. This is a major cause of morbidity including pancreatitis (7\%), cholangitis $(26 \%)$, stent occlusion (15\%), postoperative wound infection (13\%) (44) which can delay surgery or chemotherapy (45). Thus a proportion of an upfront surgery group can avoid this intervention and proceed directly to surgery where benefits are clear; however in a neoadjuvant pathway every jaundiced patient will need to undergo biliary drainage. Whilst self expanding metal stents can reduce complications they are still associated with more complications than up front surgery (46), and often patients still undergo placement of a plastic stent (47).

Delays to treatment, particularly in the setting of RPC may once again alter the resectability of disease missing a potential 'window of opportunity (48)' and early data suggests fast-tracking to surgery in jaundiced RPC to avoid cholangitis also improves resection rates (47) and possibly survival (49). Though it must be recognised that those where resectability is at risk may represent highly aggressive tumour biology that may have limited benefit from surgery at all. The additional hurdle NAT poses may compound the failure to reach resection.

\section{Emerging and ongoing trials}

There is a severe paucity of level 1 evidence to guide NAT for RPC and most current recommendations are derived from largely non-randomised data (50) and trials that include BRPC and LAPC (7). There is now emerging randomised data comparing US $v s$. NAT for specifically RPC, however, they are yet to reach final publication (Table 3).

Preliminary data from the "Randomized phase II/III trial of neoadjuvant chemotherapy with gemcitabine and S-1 versus upfront surgery for resectable pancreatic cancer (Prep-02/JSAP-05)", comparing neoadjuvant gemcitabine and $\mathrm{S} 1$ vs. upfront surgery was recently published. Here 364 patients were recruited in 57 centers. Two cycles of gemcitabine and oral S-1was administered in the NAT arm 
Table 3 Summary of emerging and ongoing trials comparing neoadjuvant therapy $v s$. upfront resection and adjuvant therapy for resectable pancreatic cancer

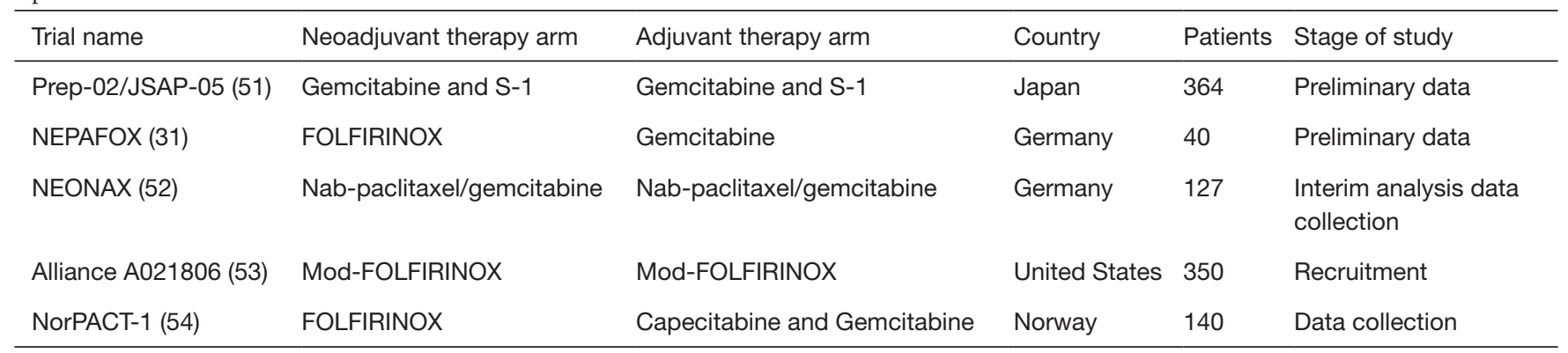

NAT, neoadjuvant therapy; AT, adjuvant therapy; mod, modified; FOLFIRINOX, folinic acid, 5-fluorouracil, irinotecan, and oxaliplatin.

then adjuvant S-1 for 6 months in both arms. Investigators observed an improvement in median overall survival among patients in the NAT arm (36.7 vs. 26.6 months, $\mathrm{P}=0.015$ ) (51). Investigators further noted comparable resection rates, $\mathrm{R} 0$ resection rates, and morbidity between the two arms. This is in contrast to the preliminary results of the aforementioned NEPAFOX RCT (31). The final publications for both of these trials are eagerly awaited.

The interim analysis of the German NEONAX trial (NCT02047513) (52) have also been published. This is a phase II trial run by the Working Group for Medical Oncology from the German Cancer Society comparing neoadjuvant nab-paclitaxel/gemcitabine $v$ s. the same combination in the adjuvant setting for RPC. Following the recruitment of 127 patients, investigators observed progression of disease seen at the time of surgery in $8 \%$ of patients in the NAT arm. Overall survival outcomes are not yet confirmed.

Two further ongoing trials remain in the recruitment and data collection phase at present: The Alliance A021806 Trial (Testing the Use of the Usual Chemotherapy Before and After Surgery for Removable Pancreatic Cancer NCT04340141) (53) by the National Cancer Institute (NCI) comparing neoadjuvant modified FOLFIRINOX with adjuvant modified FOLFIRINOX in RPC and the Nordic Pancreatic Cancer Trial (NorPACT-1, NCT02919787) comparing neoadjuvant FOLFIRINOX vs. adjuvant capecitabine and gemcitabine in RPC (54). The results of these trial will provide the necessary level 1 comparative data to guide further recommendations.

\section{Conclusions}

As NAT is becoming the standard of care for BRPC and $\mathrm{LAPC}$, its role in RPC remains a topic of increasing interest. Though we have observed improved R0 resection rates, this has yet to translate to a robust improvement in overall survival. Furthermore, it is becoming increasingly evident that a proportion of patients that have disease progression during the period of NAT may transition from once an RPC to an un RPC and we have yet to formulate a method to identify these patients. We eagerly await the results of ongoing trials to better guide international recommendations.

\section{Acknowledgments}

Funding: None.

\section{Footnote}

Provenance and Peer Review: This article was commissioned by the Guest Editor (Savio George Barreto) for the series "Unresolved Issues in Pancreatic Cancer" published in Chinese Clinical Oncology. The article has undergone external peer review.

Reporting Checklist: The authors have completed the Narrative Review reporting checklist. Available at https:// cco.amegroups.com/article/view/10.21037/cco-21-161/rc

Conflicts of Interest: All authors have completed the ICMJE uniform disclosure form (available at https://cco. amegroups.com/article/view/10.21037/cco-21-161/coif). The series "Unresolved Issues in Pancreatic Cancer" was commissioned by the editorial office without any funding or sponsorship. The authors have no other conflicts of interest to declare.

Ethical Statement: The authors are accountable for all 
aspects of the work in ensuring that questions related to the accuracy or integrity of any part of the work are appropriately investigated and resolved.

Open Access Statement: This is an Open Access article distributed in accordance with the Creative Commons Attribution-NonCommercial-NoDerivs 4.0 International License (CC BY-NC-ND 4.0), which permits the noncommercial replication and distribution of the article with the strict proviso that no changes or edits are made and the original work is properly cited (including links to both the formal publication through the relevant DOI and the license). See: https://creativecommons.org/licenses/by-nc-nd/4.0/.

\section{References}

1. Rahib L, Smith BD, Aizenberg R, et al. Projecting cancer incidence and deaths to 2030: the unexpected burden of thyroid, liver, and pancreas cancers in the United States. Cancer Res 2014;74:2913-21.

2. Seufferlein T, Hammel P, Delpero JR, et al. Optimizing the management of locally advanced pancreatic cancer with a focus on induction chemotherapy: Expert opinion based on a review of current evidence. Cancer Treat Rev 2019;77:1-10.

3. Siegel RL, Miller KD, Fuchs HE, et al. Cancer Statistics, 2021. CA Cancer J Clin 2021;71:7-33.

4. Conroy T, Hammel P, Hebbar M, et al. FOLFIRINOX or Gemcitabine as Adjuvant Therapy for Pancreatic Cancer. N Engl J Med 2018;379:2395-406.

5. Oettle H, Post S, Neuhaus P, et al. Adjuvant chemotherapy with gemcitabine vs observation in patients undergoing curative-intent resection of pancreatic cancer: a randomized controlled trial. JAMA 2007;297:267-77.

6. Bilimoria KY, Bentrem DJ, Ko CY, et al. Multimodality therapy for pancreatic cancer in the U.S. : utilization, outcomes, and the effect of hospital volume. Cancer 2007;110:1227-34.

7. Ratnayake B, Savastyuk AY, Nayar M, et al. Recurrence Patterns for Pancreatic Ductal Adenocarcinoma after Upfront Resection Versus Resection Following Neoadjuvant Therapy: A Comprehensive Meta-Analysis. J Clin Med 2020;9:2132.

8. Strobel O, Hank T, Hinz U, et al. Pancreatic Cancer Surgery: The New R-status Counts. Ann Surg 2017;265:565-73.

9. Tummers WS, Groen JV, Sibinga Mulder BG, et al. Impact of resection margin status on recurrence and survival in pancreatic cancer surgery. Br J Surg 2019;106:1055-65.

10. Ghaneh P, Kleeff J, Halloran CM, et al. The Impact of Positive Resection Margins on Survival and Recurrence Following Resection and Adjuvant Chemotherapy for Pancreatic Ductal Adenocarcinoma. Ann Surg 2019;269:520-9.

11. Isaji S, Mizuno S, Windsor JA, et al. International consensus on definition and criteria of borderline resectable pancreatic ductal adenocarcinoma 2017. Pancreatology 2018;18:2-11.

12. Varadhachary GR, Tamm EP, Abbruzzese JL, et al. Borderline resectable pancreatic cancer: definitions, management, and role of preoperative therapy. Ann Surg Oncol 2006;13:1035-46.

13. Katz MH, Pisters PW, Evans DB, et al. Borderline resectable pancreatic cancer: the importance of this emerging stage of disease. J Am Coll Surg 2008;206:83346; discussion 846-8.

14. Callery MP, Chang KJ, Fishman EK, et al. Pretreatment assessment of resectable and borderline resectable pancreatic cancer: expert consensus statement. Ann Surg Oncol 2009;16:1727-33.

15. NCCN. Pancreatic Adenocarcinoma (Version 2.2016). 2016. Available online: http://www.nccn.org/professionals/ physician_gls/pdf/pancreatic.pdf. Accessed 12/10/21.

16. Katz MH, Marsh R, Herman JM, et al. Borderline resectable pancreatic cancer: need for standardization and methods for optimal clinical trial design. Ann Surg Oncol 2013;20:2787-95.

17. Assifi MM, Lu X, Eibl G, et al. Neoadjuvant therapy in pancreatic adenocarcinoma: a meta-analysis of phase II trials. Surgery 2011;150:466-73.

18. Groot VP, Gemenetzis G, Blair AB, et al. Defining and Predicting Early Recurrence in 957 Patients With Resected Pancreatic Ductal Adenocarcinoma. Ann Surg 2019;269:1154-62.

19. Groot VP, Rezaee N, Wu W, et al. Patterns, Timing, and Predictors of Recurrence Following Pancreatectomy for Pancreatic Ductal Adenocarcinoma. Ann Surg 2018;267:936-45.

20. Van den Broeck A, Sergeant G, Ectors N, et al. Patterns of recurrence after curative resection of pancreatic ductal adenocarcinoma. Eur J Surg Oncol 2009;35:600-4.

21. Paik KY, Choi SH, Heo JS, et al. Analysis of liver metastasis after resection for pancreatic ductal adenocarcinoma. World Journal of Gastrointestinal Oncology 2012;4:109-14.

22. Haeno H, Gonen M, Davis MB, et al. Computational 
modeling of pancreatic cancer reveals kinetics of metastasis suggesting optimum treatment strategies. Cell 2012;148:362-75.

23. Neoptolemos JP, Stocken DD, Friess H, et al. A randomized trial of chemoradiotherapy and chemotherapy after resection of pancreatic cancer. N Engl J Med 2004;350:1200-10.

24. Neoptolemos JP, Palmer DH, Ghaneh P, et al. Comparison of adjuvant gemcitabine and capecitabine with gemcitabine monotherapy in patients with resected pancreatic cancer (ESPAC-4): a multicentre, open-label, randomised, phase 3 trial. Lancet 2017;389:1011-24.

25. Park W, Chawla A, O'Reilly EM. Pancreatic Cancer: A Review. JAMA 2021;326:851-62.

26. Asare EA, Evans DB, Erickson BA, et al. Neoadjuvant treatment sequencing adds value to the care of patients with operable pancreatic cancer. J Surg Oncol 2016;114:291-5.

27. Tajima H, Ohta T, Okazaki M, et al. Neoadjuvant chemotherapy with gemcitabine-based regimens improves the prognosis of node positive resectable pancreatic head cancer. Mol Clin Oncol 2019;11:157-66.

28. Kaissis G, Braren R. Pancreatic cancer detection and characterization-state of the art cross-sectional imaging and imaging data analysis. Transl Gastroenterol Hepatol 2019;4:35.

29. Kim HY, Lee YJ, Chang W, et al. Tumor resectability and response on CT following neoadjuvant therapy for pancreatic cancer: inter-observer agreement study. Eur Radiol 2022. [Epub ahead of print].

30. Van Tienhoven G, Versteijne E, Suker M, et al. Preoperative chemoradiotherapy versus immediate surgery for resectable and borderline resectable pancreatic cancer (PREOPANC-1): a randomized, controlled, multicenter phase III trial. J Clin Oncol 2018;36:LBA4002.

31. Hozaeel W, Pauligk C, Homann N, et al. Randomized multicenter phase II/III study with adjuvant gemcitabine versus neoadjuvant/adjuvant FOLFIRINOX in resectable pancreatic cancer: the NEPAFOX trial. J Clin Oncol 2015;33:suppl; abstr TPS4152.

32. Reni M, Balzano G, Zanon S, et al. Safety and efficacy of preoperative or postoperative chemotherapy for resectable pancreatic adenocarcinoma (PACT-15): a randomised, open-label, phase 2-3 trial. Lancet Gastroenterol Hepatol 2018;3:413-23.

33. Ghaneh P, Palmer DH, Cicconi S, et al. ESPAC-5F: Fourarm, prospective, multicenter, international randomized phase II trial of immediate surgery compared with neoadjuvant gemcitabine plus capecitabine (GEMCAP) or FOLFIRINOX or chemoradiotherapy (CRT) in patients with borderline resectable pancreatic cancer. J Clin Oncol 2020;38:4505.

34. Sohal D, Duong MT, Ahmad SA, et al. SWOG S1505: Results of perioperative chemotherapy (peri-op CTx) with mfolfirinox versus gemcitabine/nab-paclitaxel (Gem/nabP) for resectable pancreatic ductal adenocarcinoma (PDA). J Clin Oncol 2020;38:4504.

35. Golcher H, Brunner TB, Witzigmann H, et al. Neoadjuvant chemoradiation therapy with gemcitabine/ cisplatin and surgery versus immediate surgery in resectable pancreatic cancer. Strahlentherapie und Onkologie 2015;191:7-16.

36. Casadei R, Di Marco M, Ricci C, et al. Neoadjuvant Chemoradiotherapy and Surgery Versus Surgery Alone in Resectable Pancreatic Cancer: A Single-Center Prospective, Randomized, Controlled Trial Which Failed to Achieve Accrual Targets. J Gastrointest Surg 2015;19:1802-12.

37. Ren X, Wei X, Ding Y, et al. Comparison of neoadjuvant therapy and upfront surgery in resectable pancreatic cancer: a meta-analysis and systematic review. Onco Targets Ther 2019;12:733-44.

38. Lee YS, Lee JC, Yang SY, et al. Neoadjuvant therapy versus upfront surgery in resectable pancreatic cancer according to intention-to-treat and per-protocol analysis: A systematic review and meta-analysis. Sci Rep 2019;9:15662.

39. Cloyd JM, Heh V, Pawlik TM, et al. Neoadjuvant Therapy for Resectable and Borderline Resectable Pancreatic Cancer: A Meta-Analysis of Randomized Controlled Trials. J Clin Med 2020;9:1129.

40. Allum WH, Stenning SP, Bancewicz J, et al. Long-term results of a randomized trial of surgery with or without preoperative chemotherapy in esophageal cancer. J Clin Oncol 2009;27:5062-7.

41. Ychou M, Boige V, Pignon JP, et al. Perioperative chemotherapy compared with surgery alone for resectable gastroesophageal adenocarcinoma: an FNCLCC and FFCD multicenter phase III trial. J Clin Oncol 2011;29:1715-21.

42. Sebag-Montefiore D, Stephens RJ, Steele R, et al. Preoperative radiotherapy versus selective postoperative chemoradiotherapy in patients with rectal cancer (MRC CR07 and NCIC-CTG C016): a multicentre, randomised trial. Lancet 2009;373:811-20.

43. Conroy T, Desseigne F, Ychou M, et al. FOLFIRINOX 
versus gemcitabine for metastatic pancreatic cancer. $\mathrm{N}$

Engl J Med 2011;364:1817-25.

44. van der Gaag NA, Rauws EA, van Eijck CH, et al. Preoperative biliary drainage for cancer of the head of the pancreas. N Engl J Med 2010;362:129-37.

45. Eshuis WJ, van der Gaag NA, Rauws EA, et al. Therapeutic delay and survival after surgery for cancer of the pancreatic head with or without preoperative biliary drainage. Ann Surg 2010;252:840-9.

46. Tol JA, van Hooft JE, Timmer R, et al. Metal or plastic stents for preoperative biliary drainage in resectable pancreatic cancer. Gut 2016;65:1981-7.

47. Roberts KJ, Prasad P, Steele Y, et al. A reduced time to surgery within a 'fast track' pathway for periampullary malignancy is associated with an increased rate of pancreatoduodenectomy. HPB (Oxford) 2017;19:713-20.

48. Del Chiaro M, Søreide K. Trials and tribulations of neoadjuvant therapy in pancreatic cancer. Br J Surg 2018;105:1387-9.

49. Pande R, Hodson J, Marudanayagam R, et al. Survival Advantage of Upfront Surgery for Pancreatic Head Cancer Without Preoperative Biliary Drainage. Front Oncol 2020;10:526514.

Cite this article as: Ratnayake CBB, Roberts KJ, Pandanaboyana S. Upfront surgery vs. neoadjuvant therapy for resectable pancreatic cancer: a narrative review of available evidence. Chin Clin Oncol 2022;11(1):2. doi: 10.21037/cco-21-161
50. Moutardier V, Turrini O, Huiart L, et al. A reappraisal of preoperative chemoradiation for localized pancreatic head ductal adenocarcinoma in a 5-year single-institution experience. J Gastrointest Surg 2004;8:502-10.

51. Motoi F, Kosuge T, Ueno H, et al. Randomized phase II/ III trial of neoadjuvant chemotherapy with gemcitabine and S-1 versus upfront surgery for resectable pancreatic cancer (Prep-02/JSAP05). Jpn J Clin Oncol 2019;49:190-4.

52. Uhl W, Ettrich TJ, Reinacher-Schick AC, et al. NEONAX trial: Neoadjuvant plus adjuvant or only adjuvant nabpaclitaxel plus gemcitabine for resectable pancreatic cancer, a phase II study of the AIO pancreatic cancer group (AIO-PAK-0313) - Safety interim analysis. J Clin Oncol 2019;37:4128.

53. National Cancer Institute. Clinical Trial: Testing the Use of the Usual Chemotherapy Before and After Surgery for Removable Pancreatic Cancer. 2020. Available online: https://clinicaltrials.gov/ct2/show/NCT04340141

54. Labori KJ, Lassen K, Hoem D, et al. Neoadjuvant chemotherapy versus surgery first for resectable pancreatic cancer (Norwegian Pancreatic Cancer Trial - 1 (NorPACT-1)) - study protocol for a national multicentre randomized controlled trial. BMC Surg 2017;17:94. 